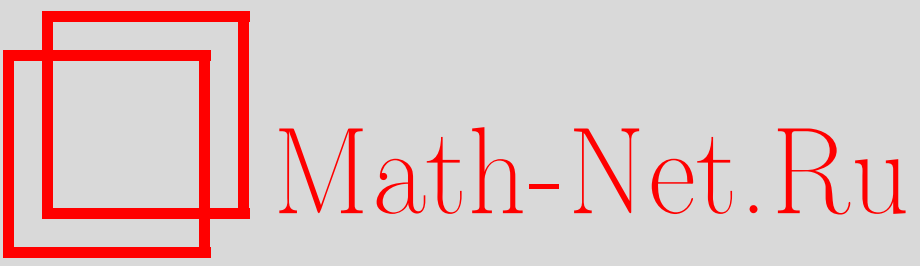

О. В. Камловский, А. С. Кузьмин, Распределение элементов на циклах линейных рекуррентных последовательностей над кольцами Галуа, УМH, 1998, том 53, выпуск 2, 149-150

DOI: https://doi.org/10.4213/rm38

Использование Общероссийского математического портала Math-Net.Ru подразумевает, что вы прочитали и согласны с пользовательским соглашением

http://www.mathnet.ru/rus/agreement

Параметры загрузки:

IP : 35.173 .137 .237

26 апреля 2023 г., 12:40:08 


\title{
РАСПРЕДЕЛЕНИЕ ЭЛЕМЕНТОВ НА ЦИКЛАХ ЛИНЕЙНЫХ РЕКУРРЕНТНЫХ ПОСЛЕДОВАТЕЛЬНОСТЕЙ НАД КОЛЬЦАМИ ГАЛУА
}

\author{
О.В. КАмловСКиЙ, А. С. Кузьмин
}

Распределение элементов в линейных рекуррентных последовательностях (ЛРП) над кольцами Галуа изучается в работах [1]-[4], [6], [9]-[12]. В данной работе изучаются частоты появления $r$-грамм на циклах ЛРП над кольцами Галуа. При условии, что характеристический многочлен ЛРП является многочленом Галуа, получены оценки частот появления $r$-грамм на цикле ЛРП. Данные оценки улучшают резултаты работ [2] и [3].

Пусть $p$ - простое число, а $t$ и $n$ - натуральные числа, $R=G R\left(p^{t n}, p^{n}\right)$ - кольо Галуа характеристики $p^{n}$ из $p^{t n}$ элементов [5], [7], $f(x)$ - многочлен над кольцом $R$ степени $m, m>1$. Всюду далее будем считать, что многочлен $f(x)$ является многочленом Галуа над кольцом $R$, т.е. образ $\bar{f}(x)$ многочлена $f(x)$ при действии естественного эпиморфизма $R[x] \longrightarrow \bar{R}[x]$, где $\bar{R}=R / p R$, является неприводимым многочленом над полем $\bar{R}$. Как показано в работе [8], период $T(f)$ многочлена $f(x)$ равен $\left(q^{m}-1\right) p^{\nu} / d$, где $q=p^{t}, d$-некоторый делитель числа $q^{m}-1$, а $\nu$-целое неотрицательное число, удовлетворяющее условиям $0 \leqslant \nu \leqslant n-1$. Через $\alpha$ обозначим корень многочлена $f(x)$ в расширении $S=G R\left(q^{m n}, p^{n}\right)$ кольца $R$. Пусть $u=(u(i))_{i=0}^{\infty}$ ЛРП над колњцом $R$ с характеристическим многочленом $f(x)$, а $T(u)$ - ее период. ЛРП $u$, для которой выполнены условия $T(u)=T(f)=\left(q^{m}-1\right) p^{n-1}$, будем назьвать ЛРП максимального периода над кольцом $R$. Пусть $r$ - произвольное натуральное число, $s_{1}, s_{2}, \ldots, s_{r}$ - целье неотрицательные числа, $z_{1}, z_{2}, \ldots, z_{r}$-элементы колцц $R, \bar{z}=\left(z_{1}, z_{2}, \ldots, z_{r}\right), \bar{s}=\left(s_{1}, s_{2}, \ldots, s_{r}\right)$. Через $N_{\bar{z}}^{\bar{s}}(u)$ обозначим количество целых чисел $i \in \overline{0, T(u)-1}$, удовлетворяющих равенствам $u\left(i+s_{j}\right)=z_{j}, j \in \overline{1, r}$. Будем изучать отклонение частот $N_{\bar{z}}^{\bar{s}}(u)$ от величины $\omega_{\bar{z}}(u)$, которая определена следующими равенствами:

$$
\omega_{\bar{z}}(u)= \begin{cases}\frac{q^{m-n r}-1}{q^{m}-1} T(u), & \text { если } \bar{z}=\overline{0}, \\ \frac{q^{m-n r}}{q^{m}-1} T(u), & \text { если } \bar{z} \neq \overline{0} .\end{cases}
$$

Величины $\omega_{\bar{z}}(u) / T(u)$ и $1 / q^{n r}$ - "идеальная" относительная частота появления $r$-граммы, связаны соотношением $\left|\omega_{\bar{z}}(u) / T(u)-1 / q^{n r}\right|<p^{n-1} / T(u)$. Поэтому при фиксированных значениях $q, n, r$ и при достаточно больших значениях периода $T(u)$ ЛРП $u$ величины $\omega_{\bar{z}}(u) / T(u)$ и $1 / q^{n r}$ можно считать равными.

Теорема 1. Пусть среди әлементов $u(0), u(1), \ldots, u(m-1)$ есть хотя бъ один обратимый элемент кольца $R$ и система әлементов $\alpha^{s_{1}}, \alpha^{s_{2}}, \ldots, \alpha^{s_{r}}$ линейно независима над кольцом $R$. Тогда справедливо неравенство:

$$
\left|N_{\bar{z}}^{\bar{s}}(u)-\omega_{\bar{z}}(u)\right| \leqslant \frac{p^{\nu}\left(\left(q^{n r} p^{n}-1\right)\left(q^{r}-1\right) d-\left(q^{r} p-1\right)\left(q^{n r}-1\right)\right)}{q^{n r} d\left(q^{r} p-1\right)} q^{m / 2} .
$$

В частности, в условиях теоремы всегда выполнено неравенство:

$$
\left|N_{\bar{z}}^{\bar{s}}(u)-\omega_{\bar{z}}(u)\right| \leqslant p^{\nu+n-1} q^{m / 2} .
$$

Из результата (1) следуют верхняя и нижняя оценки частот $N \overline{\bar{z}}(u)$. При условии

$$
T(u) \geqslant p^{2(n-1)} q^{\frac{m}{2}+n r}
$$

эти оценки частоты $N \frac{\bar{s}}{\bar{z}}(u)$ будут нетривиальными для всех $r$-грамм $\bar{z} \in R^{r}$.

В работе [3] изучаются частотные характеристики ЛРП максимального периода над кольцом $\mathbb{Z} / p^{2}$. При этом для модуля отклонения частот появления элементов на цикле ЛРП от величины $p^{m-1}$ получена верхняя оценка $p^{\frac{3 m}{4}+1}$. Правая часть неравенства (1) в этом случае равна 
$(p-1)^{2} p^{\frac{m}{2}}$, что существенно улучшает эту оценку. В работе [2] получены нижние оценки частот появления элементов на циклах ЛРП максимального периода над кольцм $\mathbb{Z} / p^{n}$. Результат (1) улучшает эти оценки при условии $m \geqslant 4 n$. Кроме того, в некоторых случаях резулштат (1) улучшает известные оценки частот появления $r$-грамм на циклах ЛРП над конечньми полями [6], [9]-[12].

Из теоремы 1 можно получить условия, при которых на цикле ЛРП $u$ заведомо появятся все $r$-граммы элементов кольца $R$.

СЛЕДСТвИЕ 2. Пусть в условиях теоремы 1 дополнительно выполняется хотя бы одно из условий

1) $T(u) \geqslant p^{2(n-1)}\left(q^{n r}-1\right) q^{m / 2}$,

2) $u$ - ЛРП максимального периода над кольцом $R$ u $m \geqslant 2(t n r+n-1) / t$.

Тогда среди г-грамм $\left(u\left(i+s_{1}\right), u\left(i+s_{2}\right), \ldots, u\left(i+s_{r}\right)\right), i \in \overline{0, T(u)-1}$, появятся все әлементы множества $R^{r}$.

Приведем теперь нижнюю оценку для модуля отклонения частот появления $r$-грамм на цикле ЛРП $u$ максимального периода от величины $T(u) / q^{n r}$.

Теорема 3. Пусть период $T(f)$ многочлена $f(x)$ равен $\left(q^{m}-1\right) p^{n-1}$ и система әлементов $\alpha^{s_{1}}, \alpha^{s_{2}}, \ldots, \alpha^{s_{r}}$ линейно независима над кольцом $R$. Тогда найдутся ЛРП $u$ максимального периода с характеристическим многочленом $f(x)$ u $r$-грамма $\bar{z} \in R^{r}$ такие, что:

əəe

$$
\left|N_{\bar{z}}^{\bar{s}}(u)-\frac{T(u)}{q^{n r}}\right|>C(n, r, q) q^{m / 2},
$$

$$
C(n, r, q)= \begin{cases}\left(\frac{(p-1)^{2}(n-1)}{p^{3}}\right)^{1 / 2}, & \text { если } p=q, r=1, n>1, \\ \left(\frac{\left(q^{r}-1\right)(p-1) p^{n-2}}{q^{(n+1) r}}\right)^{1 / 2}, & \text { если } q^{r}>p, n>1 .\end{cases}
$$

Из последнего результата следует, что если колцо $R$ не является полем, то в классе всех ЛРП максимального периода с заданньм характеристическим многочленом $f(x)$ всегда найдется ЛРП, у которой частотные характеристики отличны от "идеальных" значений. Более того, этот результат показывает, что множитель $q^{m / 2}$ в оценке $(1)$, характеризующий зависимость изучаемой разности от степени $m$ характеристического многочлена ЛРП, по-видимому, нельзя уменьшить.

\section{СПИСОК ЛИТЕРАТУРЫ}

[1] Коробов Н. М. // Докл. АН СССР. 1953. Т. 88. № 4. С. 603-606. [2] Кузьмин А. С. // УMH. 1992. T. 47. №6. C. 213-214. [3] Kuzmin A. S., Nechaev A. A. // Proceedings of the IV Internat. Workshop "Algebr. and Combinator. Coding Theory". Sofia: Zakrila-Novgorod, 1994. Р. 132-136. [4] Лидл Р., Нидеррайтер Г. Конечные поля. Т. 1, 2. М.: Мир, 1988. [5] McDonald B. R. Finite rings with identity. New York: Dekker, 1974. [6] McEliece R. J. Irreducible cyclic codes and Gauss sums // Combinatorics / ed. M. Hall, Jr. and J.H. van Lint, 1975. Р. 185-202. [7] Нечаев А. А. // Дискретн. матем. 1989. Т. 1. № 4. С. 123-139. [8] Нечаев А. А. // Матем. сб. 1993. Т. 184. № 3. С. 21-56. [9] Нечаев В. И. // Матем. заметки. 1972. T. 11. № 5. C. 597-607. [10] Niederreiter H. // Probl. Control Inform. Theory. 1986. V. 15. № 1. Р. 19-34. [11] Сидельников В. М. // Дискретн. матем. 1991. Т. 3. № 2. С. 87-95. [12] Шпарлинский И. Е. // Проблемы передачи информации. 1989. Т. 25. № 2. С. 46-53. 